\title{
About visualization of the aroma of fruits
}

\author{
Alexander A. Kharlamov", Hugh D. Burrows \\ Department of Chemistry, University of Coimbra, Coimbra 3049, Portugal \\ Received Received 23 October 2000; ; accepted accepted 20 December 2000
}

\begin{abstract}
Fluorimetry has been used to detect the luminescence in vivo of native organic volatiles emitted from the surface of various fruits. This luminescence near the surface of fruits was visualized in the visible spectral region as color photo-images using different excitations. While the origin of this luminescence has not yet been confirmed, the results demonstrate that fluorimetry of natural aroma emission is a very promising method for studying biochemical environment of fruits that may be extended to other living systems. (C) 2001 Elsevier Science B.V. All rights reserved.
\end{abstract}

Keywords: Luminescence; Volatile compounds; Fluorimetry of aroma emission; Living systems

\section{Introduction}

This short note extends our recent research into light induced emission from various fruits [1] to in vivo visualization of this luminescence near the fruit surface. This approach uses the ability of volatile compounds produced by fruit to luminesce in the vicinity of the fruit surface when irradiated by a laser beam. In a previous study using laser fluorimetry it was suggested that this light emission could be attributed to a gaseous atmosphere of native organic volatiles emitted from the surface of fruits. While the origin of this emission has not yet been confirmed, its existence allows minute amounts of substance to be successfully detected and characterized. Moreover, the spectroscopic capacity of such a method allows for a reliable discrimination between different physiological states of living species, while the optical focusing and scanning of excitation light permits mapping of emitting regions as small as few microns of the plant surface. As was shown in [1], most of organic volatiles can luminesce effectively during the emission through the skin of fruit, which makes such a remote "artificial nose" a very general approach and potentially an extremely powerful tool for the investigation of the metabolism not only of plants, but also of animals, including humans, and therefore provides many biomedical applications.

\footnotetext{
* Corresponding author. Permanent address: Institute of Biochemical Physics, RAS, Kosygin st. 4, 117334 Moscow, Russia.

E-mail address: akh@ci.uc.pt (A.A. Kharlamov).
}

Until recently, the analysis of aromas has been based on methods for the extraction, separation and identification of volatile organic compounds by high resolution gas or liquid chromatography hyphenated with mass-spectrometry and/or infra-red spectroscopy. However, in any isolation method, the final extract is never completely faithful to the true aroma. It has been reported, based on isolation methods, that, for example, fruits produce complex mixtures of volatiles with more than 200 identified compounds [2-5]. The compounds responsible for a particular aroma are normally accompanied by many other volatile components. It is generally assumed that only some of these compounds provide most of the aroma associated with a fruit.

Recent interest in the real time detection of volatile organic compounds brings together researchers from different backgrounds. Such use of advanced interdisciplinary areas carries significant potential both in fundamental science as well as in new technology developments. An electronic sensing system (electronic sniffer) based on aroma sensor array has already been developed. The sensor array responds to the presence of specific aromas by sensor resistance changes and this can be used for real time monitoring of the total concentration of authentic volatiles [6]. A new powerful method based on proton-transferreaction-mass-spectrometry has recently been introduced for trace gas detection in real time [7,8]. This was successfully applied to food control, pollution, environmental problems [9], and has quite recently, been used in real time monitoring of organic volatiles emitted from fruits during aging [10]. It has been demonstrated that the concentrations of volatiles emitted from fruits can be related to a sequence 
of the compounds: methanol, ethanol, acetaldehyde, methyl acetate, acetone, acetic acid, ethyl acetate, mixture of ketones and aldehydes, and esters. As clearly shown in [10], the aging processes in fruits are related directly to the emission of these organic compounds.

Because of its high sensitivity, fluorimetry has been used increasingly as a diagnostic tool in biomedical practice, biochemistry, pharmacology, organic chemistry, and related fields in which the main objectives are organic trace analysis or identification of chemical species. Fluorimetry has been used to detect the luminescence of native organic volatiles emitted at the surface of fruits [1]. We report in vivo visualization of the luminescence from the aroma of fruits in the visible region. Our results shows that luminescence spectroscopy of the organic volatiles is a very promising method for monitoring of chemical evolution in living systems.

\section{Experimental}

Fruits (apples, pears, and kiwi) from various origins were all purchased locally and were kept in individual open containers at room temperature under normal atmosphere. In these preliminary studies no information was available on when the fruit was picked.

Photoluminescence spectra were measured at room temperature with a Jobin Yvon-SPEX Fluorolog-3 spectrofluorimeter with a $450 \mathrm{~W}$ Xenon arc lamp as excitation source. Excitation wavelengths $( \pm 2 \mathrm{~nm})$ were isolated by appropriate monochromators with filter combinations. Luminescence measurements were performed in an unpolarized configuration using the $90^{\circ}$-scattering geometry, with the excitation light at right angles to the sample surface. The spectral components were calculated from the data using Microcal Origin-5 (Microcal Software, Inc.). Photoluminescence near the fruit was visualized using a PC-camera (Logitech QuickCamPro) and analysis performed by the UTHSCSA ImageTool-2.00 program.

\section{Results and discussion}

Luminescence spectra from fruits were performed using different excitation wavelengths in the range $\lambda_{\mathrm{exc}}=$ 250-600 nm, as described in experimental session. Fig. 1 represents the typical spectra of fruit (an apple) measured using excitation by Xenon lamp. In the $650-800 \mathrm{~nm}$ region (curves 3,4,5), the peak at $681 \mathrm{~nm}$ and relatively weak band at 700-730 nm observed for various excitation wavelengths, were assigned to luminescence, as was evident from the initial results of the Stokes and the anti-Stokes measurements [1]. On decreasing the excitation wavelength down to $\lambda_{\text {exc }}=250 \mathrm{~nm}$, the intensity of the photoluminescence in the $650-800 \mathrm{~nm}$ region decreases and the spectra are dominated by a high-energy bands at $335,390,460,505,534 \mathrm{~nm}$,

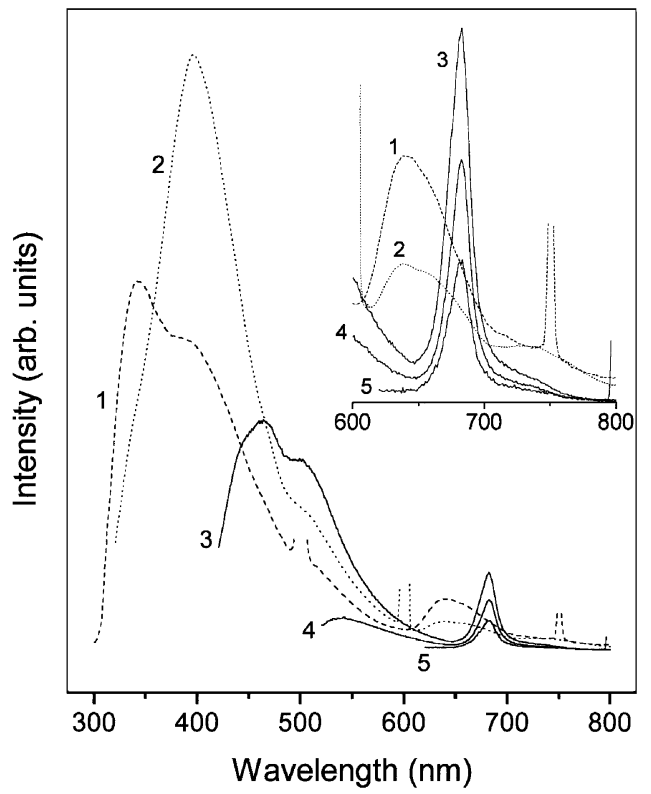

Fig. 1. 7Luminescence spectra from fruit (an apple) measured under various excitation wavelengths: spectra 1, 2, 3, 4 and 5 excited, respectively, at $\lambda_{\text {exc }}=250,300,400,500$ and $600 \mathrm{~nm}$ wavelengths (Xenon arc lamp). The insert gives the same spectra in $600-800 \mathrm{~nm}$ region in more details (peaks at 500, 600, 750 and $800 \mathrm{~nm}$ have instrument origin).

and a weak, broad band at $640 \mathrm{~nm}$ (curves 1, 2, 3). The relatively strong bands found under higher energy excitation $\left(\lambda_{\mathrm{exc}}=250,300,400 \mathrm{~nm}\right)$ were also assigned to luminescence. Alternative assignment to Raman bands is ruled out, since the energy difference between excitation and emission is not constant. The spectra obtained from different fruits have been classified into two sets: (1) very strong contributions at 330-460 and 680-685 nm, and (2) relatively weak broad bands located in the 500-640 and 700-730 nm regions.

To examine the real gas emission features, luminescence of fruits was visualized as photo-images by excitation with a $\mathrm{He}-\mathrm{Ne}$ laser beam at right angles to the sample surface and using the PC-camera. Fig. 2 (on the left) represents photoimages of an apple (top) and turnip, as a reference sample with low emission of aroma (bottom). In these images, strong red photoluminescence near surface of the apple was attributed to spectral bands in $680-700 \mathrm{~nm}$ region (curves 3, 4, 5, Fig. 1), while the intensity of luminescence near the turnip was very small. These results strongly suggest that this luminescence can be assigned to the native gas emitted from fruit.

Luminescence of the fruits was also visualized using the optical filters as color photo-images by near UV-excitation (150 W medium-pressure Mercury lamp). Luminescence from a sample was collected through the UV-filter directly onto the photo camera (Pentax, Espio-160) inside a dark room. Photo-images on the right in Fig. 2 for two fruits, apple (top) and kiwi (bottom), demonstrate the blue and the 


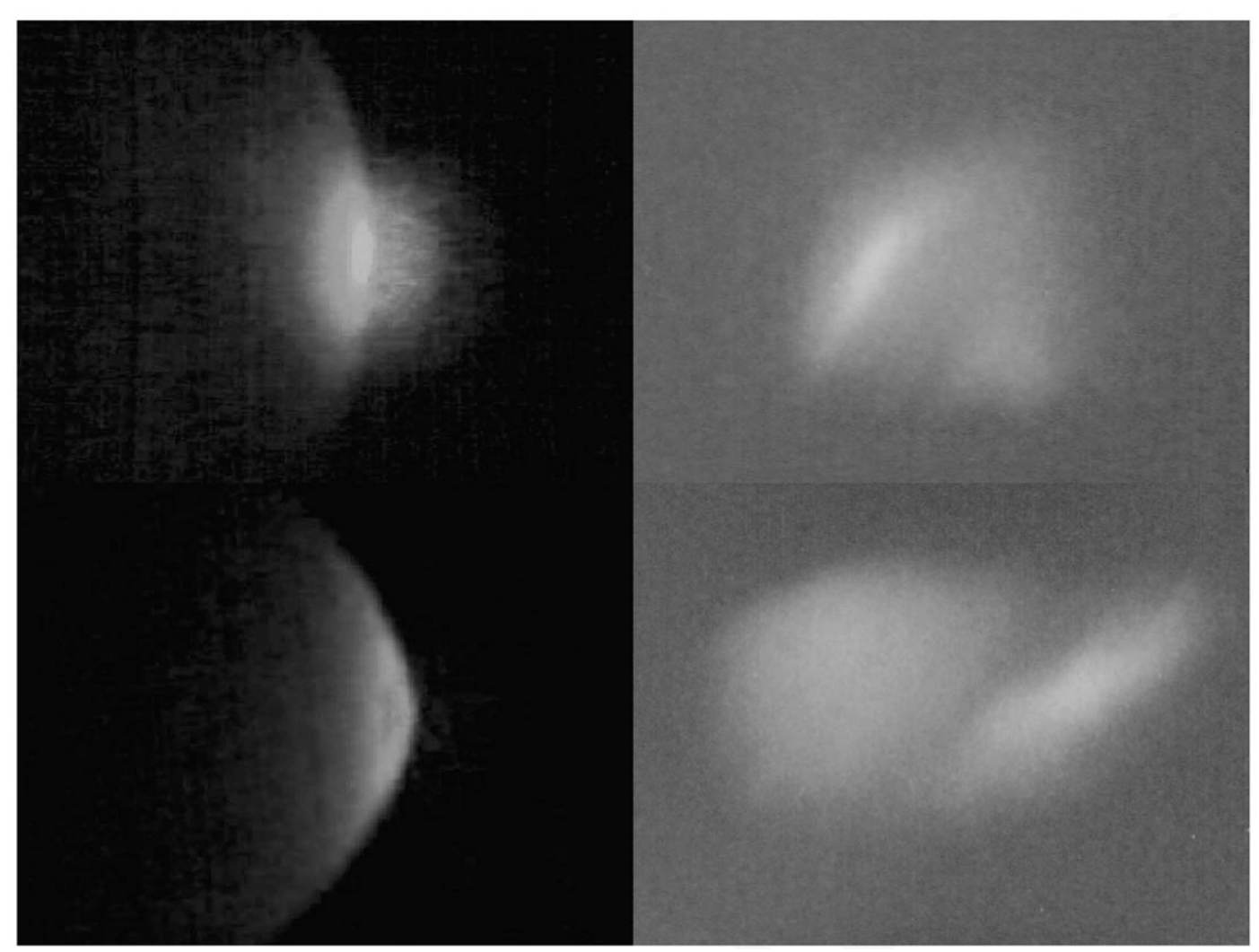

Fig. 2. Visualization of luminescence near the fruit surface: images on the left were performed with a He-Ne laser beam at right angles to the surface of an apple (top) and a turnip (bottom) and excitation power of $4 \mathrm{~mW}$ at the source; and on the right - photo-images of an apple (top) and a kiwi (bottom) demonstrate the blue and the green luminescence near surface of fruits under near UV-excitation (150 W medium-pressure Mercury lamp).

green spectral contributions that can be attributed to the photoluminescence bands observed in the visible range 460$534 \mathrm{~nm}$ (curves 1, 2, 3, Fig. 1).

We wish to emphasize that our results demonstrate a new application of luminescence spectroscopy of organic volatiles as a very promising method for studying in vivo biochemical changes in fruits, which may be extended to other living systems. The measurements presented allow an observation of the luminescence from the fruits. A number of fruits were studied, exhibiting luminescence bands attributed to organic volatiles leaving from the fruit surface. The nature of the emission processes responsible for the luminescence has yet to be determined, although it seems very likely that some higher vibrational states dominate the spectra. In our experiments, the excitation energies (1.9$4.9 \mathrm{eV}$ ) are too low to transform the volatile molecules into excited electronic states by a single-photon process. For example, the lowest excited singlet electronic state of methanol is above $6 \mathrm{eV}$ [11]. Since the molecules must be in their ground electronic states, several vibrational processes may occur. It is possible that this luminescence may be associated with the vibrations of functional groups of some volatiles, although this cannot be determined from the experiments reported here.

\section{Conclusions}

The results demonstrate that luminescence spectroscopy of organic volatiles leaving from the surface of fruit is a very promising method for the control and improvement of the quality of fruits, and that it may be extended to odours coming from other living systems. While it is well known that living systems produces many types of volatiles, there is a lack of information on the formation and emission of compounds responsible for such aromas. The real time monitoring of the volatiles emitted from fruit could give key information to the food industry for determining optimal harvesting dates and storage conditions to maintain and improve quality. In addition, in vivo identification of aromas can play a crucial role in the understanding of biosynthetic pathways of relevant components and in the identification of mechanisms that regulate these pathways at the molecular level.

\section{Acknowledgements}

The authors wish to thank the University of Coimbra and PRAXIS-XXI for their support of this research. We 
thank O. Kolosov, S. Iannotta, and A. Boschetti for helpful discussions.

\section{References}

[1] A. Kharlamov, H. Burrows, Visualization of volatiles of fruits by photoluminescence technique, Appl. Biochem. Microbiol. 37 (2001), in press.

[2] C. Mussinan, J. Walradt, Organic acids from fresh california strawberries, J. Agric. Food Chem. 23 (1975) 482-484.

[3] E. Guichard, Identification of the flavoured volatile components of the raspberry cultivar lloyd george, Sci. Des. Aliments 2 (1982) 173-185.

[4] A. Nikiforov, L. Jirovetz, A. Woidich, Evaluation of combined GC/ MS/FTIR data sets of strawberry aroma, Food Quality Preference 5 (1994) 135-137.

[5] G.W. Robertson, D.W. Griffiths, J.A. Woodford, A.N. Birch, Changes in the chemical composition of volatile released by the flowers and fruits of the red raspberry (rubus idaeus) cultivar glen prosen, Phytochemistry 38 (1995) 1175-1179.

[6] J. Simon, A. Hetzroni, B. Bordelon, G. Mills, D. Charles, Electronic sensing of volatiles for quality sorting of blueberries, J. Food Sci. 61 (1996) 967-969.

[7] A. Hansel, A. Jordan, R. Holzinger, P. Prazeller, W. Vogel, W. Lindinger, Proton-transfer-mass-spectroscopy: on-line trace gas analysis at ppt level, Int. J. Mass Spectrom. Ion Proc. 149/150 (1995) 600-619.

[8] W. Lindinger, A. Hansel, A. Jordan, On-line monitoring of volatile organic compounds at ppv levels by means of proton-transferreaction-mass spectrometer (PTR-MS). Medical application, food control and environmental research, Int. J. Mass Spectrom. Ion Proc. 173 (1998) 91-241.

[9] A. Hansel, A. Jordan, C. Warneke, R. Holzinger, W. Lindinger, Improved detection limit of the proton-transfer-reaction-mass spectrometer (PTR-MS): on-line monitoring of volatile organic compounds at mixing ratios of 10 pptv, Rapid Commun. Mass Spectrom. 12 (1998) 871-875.

[10] A. Boschetti, F. Biasioli, M. van Opbergen, C. Warneke, A. Jordan, R. Holzinger, P. Prazeller, T. Karl, A. Hansel, W. Lindinger, S. Iannotta, PTR-MS real time monitoring of the emission of volatile organic compounds during aging of berries, Postharvest Biol. Technol. 17 (1999) 143-151.

[11] S.L. Murov, I. Carmichael, G.L. Hug, Handbook of Photochemistry, 2nd Edition, Marcel Dekker, New York, 1993, p. 288.

\section{Biographies}

Alexander A. Kharlamov obtained his MSc in Physics from Moscow State University and his PhD from the Institute of Solid State Physics (RAS) in area of the Surface and Solid State Physics. Since 1983 he has been Senior Researcher at N.N. Semenov Institute of Chemical Physics and N.M. Emmanuel Institute of Biochemical Physics, RAS, and his research interests in fields involving physics of condensed matter, spectroscopy, semiconductors and thin films.

Hugh D. Burrows obtained his BSc in Chemistry from the University of London and his DPhil from the University of Sussex in the area of Physical Organic Chemistry. He has been Professor of Chemistry at the University of Coimbra since 1983, and has wide research interests in areas involving photochemistry, luminescence, polymers and surfactants. 